\title{
Tuberculose: acolhimento e informação na perspectiva da visita domiciliária
}

\author{
Tuberculosis: user embracement and information in home visits \\ Tuberculosis: acogida e información en la perspectiva de la visita domiciliaria
}

\author{
Francisco de Sales Clementino'; Francisco Arnoldo Nunes de Miranda ${ }^{I I}$
}

\begin{abstract}
RESUMO: Esta pesquisa teve como objetivo analisar a atuação dos profissionais da estratégia saúde da família nas visitas domiciliares realizadas ao doente de tuberculose. Estudo descritivo com abordangem qualitativa, realizado em janeiro de 2009, no município de Campina Grande, Paraíba, tendo como participantes do estudo 34 usuários com diagnóstico de tuberculose pulmonar e extrapulmonar. Os dados foram coletados por meio de entrevistas semiestruturadas e analisados por meio da análise de conteúdo. Os resultados apontam três categorias: visita domiciliária - a saúde bate a sua porta; sentimentos - segurança e gratidão no enfrentamento da situação; divulgação - uma questão de dispersão da informação. Concluiu-se que há um distanciamento dos profissionais de saúde da estratégia saúde da família do seu papel de educador, visto que a frequência de suas atividades educativas não condiz com a demanda apresentada pelos doentes de tuberculose.

Palavras Chaves: Tuberculose; programa saúde da família; visita domiciliar; atenção primária à saúde.
\end{abstract}

ABSTRACT: This qualitative, descriptive study examined the activities of family health strategy personnel in home visits to patients with tuberculosis in Campina Grande, Paraiba, in January 2009. Study participants were 34 users diagnosed with pulmonary and extra-pulmonary tuberculosis. Data were collected through semi-structured interviews and analyzed using content analysis. The results indicate three categories: home visits - "health knocks on your door"; feelings - security and gratitude in coping with the situation; and dissemination - a matter of spreading information. It was concluded that Family Health Strategy personnel are distanced from their role as educators, given that the frequency of their educational activities falls short of demand from tuberculosis patients.

Keywords: Tuberculosis; family health program; home visit; primary health care.

RESUMEN: Esta investigación tuvo como objetivo analizar la actuación de los profesionales de la estrategia salud de la familia en las visitas domiciliarias realizadas al enfermo de tuberculosis. Es un estudio descriptivo con abordaje cualitativo, realizado en enero de 2009, en el municipio de Campina Grande, Paraíba, que tiene como participantes del estudio 34 usuarios diagnosticados con tuberculosis pulmonar y extrapulmonar. Los datos fueron colectados por medio de entrevistas semiestructuradas y analizados a través del análisis de contenido. Los resultados apuntan hacia tres categorías: visita domiciliaria - la salud Ilama a su puerta; sentimientos - seguridad y gratitud al enfrentar de la situación; divulgación - una cuestión de dispersión de la información. Se concluye que existe un distanciamiento de los profesionales de salud de la estrategia de salud familiar de su rol de educador, visto que la frecuencia de sus actividades educativas no corresponde a la demanda presentada por los enfermos de tuberculosis.

Palabras Clave: Tuberculosis; programa salud de la familia; visita domiciliaria; atención primaria a la salud.

\section{INTRODUÇÃO}

A tuberculose (TB) é uma pandemia mundial que já afeta um terço da população do planeta. Conforme dados da Organização Mundial de Saúde, um grupo de 22 países é responsável por $80 \%$ da carga de TB no mundo.

O Brasil é o $19^{\circ}$ país do mundo em número de casos novos e o $108^{\circ}$, quando se avalia a incidência da doença. Em 2010, foram notificados 70.997 casos novos de TB, com uma taxa de incidência de $37,7 \mathrm{ca}$ sos por 100.000 habitantes. Além disso, estima-se que $14 \%$ dos doentes de TB tenham também diagnóstico de HIV. E, apesar de a TB ser uma doença curável, ainda causa a morte de 8.400 pacientes no país ${ }^{1}$.
Faz-se necessário destacar ainda as questões relacionadas às atitudes e crenças sobre o preconceito, o medo do estigma e a discriminação por parte da família, dos amigos e vizinhos, levando à restrição dos relacionamentos interpessoais de parentesco e amizade, modificando, assim, hábitos e estilo de vida²

No Brasil, a descentralização das ações de saúde vem sendo tomada como diretriz organizacional, com a implantação da Constituição Federal, sendo mencionada também em legislação infraconstitucional, a saber: Leis no 8.080 e 8.142 e Norma Operacional Básica do Sistema Único de Saúde (NOB-SUS/93), entre outros ${ }^{3}$.

IEnfermeiro. Doutor em Enfermagem pela Universidade Federal do Rio Grande do Norte. Especialista em Saúde Pública. Docente da Universidade Federal de Campina Grande, Centro de Ciências Biológicas e da Saúde, Departamento de Enfermagem. Campina Grande, Paraíba, Brasil. E-mail: clementinosales@ig.com.br. IIEnfermeiro. Doutor pela Universidade de São Paulo. Docente da Universidade Federal do Rio Grande do Norte, Departamento de Enfermagem, Programa de Pós-Graduação em Enfermagem. Natal, Rio Grande do Norte, Brasil. E-mail: farnoldo@gmail.com. 
A partir desse marco histórico, as ações de controle de tuberculose passam a ser responsabilidade de todos os municípios brasileiros, haja vista que, principalmente desde a década de 1980, a tuberculose tornou-se um grave problema de saúde pública.

Nessa perspectiva, adotam-se metas internacionais de detectar $70 \%$ dos casos novos estimados e de curar $85 \%$ dos casos detectados. No $6^{\circ}$ Objetivo de Desenvolvimento do Milênio, espera-se reduzir em $50 \%$, até 2015, a prevalência e a mortalidade por TB. Desse modo, o Ministério da Saúde coloca como uma das prioridades do pacto pela vida o fortalecimento da capacidade de resposta à $\mathrm{TB}^{2}$.

É necessário abrir espaços de diálogo com a população, gerando reflexão e problematização, que possibilitem a construção de uma relação de corresponsabilidade, favorecendo formas mais humanas e efetivas no processo de trabalho em saúde, tanto para os usuários, como para os profissionais ${ }^{1,4}$.

Dessa forma, considerando que o doente de TB se apresenta vulnerável física, emocional e socialmente, devido ao seu contexto social, na maioria das vezes desfavorável, é necessário conhecer o ambiente no qual esse indivíduo está inserido a fim de que a equipe de saúde realize uma abordagem especial para cada caso, minimizando, assim, os entraves no processo de atenção à TB5 .

Para o controle da doença, a Organização Mundial de Saúde (OMS) propõe o Tratamento Diretamente Observado (DOTS) como estratégia para atingir $85 \%$ de cura, $70 \%$ de detecção de casos e reduzir o abandono ao tratamento em $5 \%{ }^{6}$.

Desse modo, o Programa Nacional de Controle da Tuberculose conta com as estratégias da Saúde da família e de agentes comunitários de saúde (ACS). A descentralização do controle da TB para a estratégia saúde da família (ESF) consiste em ampliar o acesso dos doentes ao serviço de saúde, além de proporcionar maior adesão ao tratamento por meio do vínculo criado entre as equipes e a comunidade ${ }^{7}$.

Nesse contexto, a ESF prevê a utilização da visita domiciliária (VD) como forma de instrumentalizar os profissionais para sua inserção e o conhecimento da realidade de vida da população, bem como o estabelecimento de vínculos ${ }^{8}$. Trata-se de uma atividade realizada pelos profissionais de saúde no domicílio, com o objetivo de prestar ações e serviços para a promoção, prevenção, manutenção ou recuperação da saúde.

Entretanto, há entraves para sua concretização, como a formação dos profissionais, a cultura institucional e social e a dificuldade de extrapolar os limites do território quando necessita de um atendimento em outro ponto da rede de atenção à saúde? . As circunstâncias que corroboram para o abandono do tratamento da TB advêm, muitas vezes, de um acompanhamento deficiente, o que pode ser evitado mediante uma atitude proativa das equipes de saúde da família no cuidado ao doente de TB e sua família ${ }^{10}$.

Estudo realizado no município de Bayeux, Paraíba, aponta falhas e fragilidades no processo de trabalho da ESF, durante a VD, relatando que tal prática não é adotada regularmente, pois apenas 40,2\% das unidades de saúde a realizam ${ }^{11}$. Desse modo, os profissionais precisam estar preparados para a realização da VD com competência e eficiência, não se deixando dominar pela rotina e pela banalização da assistência, o que poderá tornar suas ações mecânicas e impessoais.

Assim, torna-se imprescindível que a equipe da saúde da família se disponha a prestar uma assistência adequada à população residente em áreas de maior risco de ocorrência de TB, atuando, portanto, para a melhoria da qualidade de vida e entendendo o doente de tuberculose como um ser único, com peculiaridades, sem perder de vista seu contexto familiar e social.

Diante dos argumentos apresentados, aponta-se a seguinte questão norteadora: Como ocorre a atuação dos profissionais da estratégia saúde da família frente ao controle da TB? Para responder a esse questionamento, foi elaborado o seguinte objetivo: analisar a atuação dos profissionais da ESF na visita domiciliar realizada ao doente de tuberculose.

Contudo, o presente estudo poderá contribuir para a organização de proposições envolvendo ações específicas para a busca da humanização da assistência ao doente de tuberculose e de seu familiar.

\section{REVISÃo De Literatura}

No âmbito mundial, a tuberculose persiste como doença negligenciada e apresenta-se como um problema de saúde pública. $\mathrm{O}$ tratamento da TB não depende apenas e especificamente da existência e gratuidade dos medicamentos e de sua eficácia farmacológica. Há de se considerar os demais elementos no processo de atenção, tais como a melhora do acesso aos serviços de saúde, a adesão e a coparticipação da pessoa com tuberculose e da sua família ${ }^{12}$.

Dessa forma, entende-se que a porta de entrada de um sistema de saúde deve ser acessível ao usuário que, muitas vezes, recorre aos serviços de urgência pela enorme dificuldade de acesso. Isto resulta em sofrimento físico e emocional para o doente de tuberculose e seu grupo familiar, uma vez que, para garantir a acessibilidade, o sistema de saúde local deverá prover os ajustes necessários e indispensáveis à execução dos programas.

Nessa perspectiva, coloca-se como necessário, aos profissionais de saúde, transcender o entendimento da estratégia para além de garantir a ingestão da medicação, sendo indispensável conhecer também o modo de vida, a dinâmica familiar, crenças, opiniões e conhecimentos a respeito da doença e do próprio tratamento ${ }^{2,13}$. 
O que se espera, em relação ao Programa de Controle da Tuberculose (PCT), na área de abrangência do PSF, é que a unidade básica de saúde da família (UBSF) seja a porta de entrada da clientela, promova o acesso à população e preste uma atenção básica de qualidade, inclusive com o diagnóstico precoce da TB e supervisão do tratamento por intermédio da visita domiciliar.

Essa tarefa atende às necessidades específicas das ações em tuberculose, uma vez que o diagnóstico e o tratamento eficiente dos doentes são a base primordial para o controle da doença ${ }^{14}$.

Dessa forma, o conhecimento das necessidades de saúde das pessoas com TB permitirá que a equipe de saúde reoriente suas práticas, visando à melhoria da assistência prestada ao doente e ao seu familiar ${ }^{15}$.

\section{Metodologia}

Estudo descritivo com abordagem qualitativa, sendo o lócus o município de Campina Grande, Paraíba. A amostra foi formada por 34 usuários, selecionados por meio do banco de dados do Sistema Nacional de Agravos de Notificação (SINAN), com diagnóstico de tuberculose pulmonar e extrapulmonar. A escolha da extensão da amostra na entrevista não foi definida por números, pois se relacionou à repetição de falas e saturação dos dados.

Elegeram-se como critérios de inclusão os casos de tuberculose com tratamento concluído, relativos às pessoas residentes na área de abrangência dos seis distritos sanitários e cadastradas no PCT. Como critérios de exclusão ficaram os casos de indivíduos residentes fora do município, aqueles notificados como suspeita, porém descartados e, ainda, os casos acompanhados e tratados exclusivamente nos centros de saúde.

Dos depoimentos dos doentes de tuberculose entrevistados, emergiram as seguintes categorias: Visita domiciliária - a saúde bate a sua porta; Sentimentos - segurança e gratidão; e Divulgação - uma questão de dispersão da informação.

Os dados foram coletados no mês de janeiro de 2009, por meio de entrevistas semiestruturadas, gravadas, transcritas e arquivadas, conforme a Resolução no 196/1996 ${ }^{16}$, aprovada pelo Comitê de Ética em Pesquisa (CEP) da Universidade Estadual da Paraíba (UEPB), sob protocolo no 0519.0.133.000.0616.

Para preservação do anonimato, os participantes foram identificados com letra $\mathrm{E}$, que corresponde aos entrevistados, seguindo a ordem numérica de participação. As entrevistas foram transcritas e analisadas pelo software Alceste 4,5 e pela análise de conteúdo de Bardin ${ }^{17}$.

\section{Resultados e Discussão}

Visita domiciliária: a saúde bate a sua porta

Alguns doentes de tuberculose demonstraram a necessidade de obter mais suportes por parte dos profissionais de saúde, destacando a visita domiciliar como um espaço de promoção da saúde e construção de vínculos entre os sujeitos envolvidos no processo.

[...] visitar as famílias pelo menos uma vez por mês; conversar para ver se combate a tuberculose. (E25)

\section{[...] visitar a gente, acompanhar direitinho. (E33)}

Pôde-se verificar que os profissionais da ESF não desenvolvem suas atividades educativas conforme os pressupostos do Ministério da Saúde, interferindo na qualidade da assistência prestada; constatou-se que o atendimento clínico ainda é predominante nas condutas dos profissionais na UBSF e/ou domicílio. De acordo com normas técnicas do Departamento de Atenção Básica do Ministério da Saúde, recomenda-se que haja, no mínimo, uma visita mensal a cada domicílio da área de atuação da estratégia saúde da família, havendo variações em função do estado de saúde de seus habitantes ${ }^{18}$.

Em relação às dificuldades da VD, é importante salientar que não adianta apenas determinar que a equipe de saúde aborde a família como uma unidade de cuidado, pois o que falta são instrumentos e instruções para os profissionais que atuam nesse ramo de assistência à saúde. Neste contexto, percebe-se, por meio da prática clínica, que, em algumas situações, a VD acontece de forma mecanizada e sem o entendimento do valioso instrumento que ela pode se constituir no processo de trabalho em saúde e, em particular, nos serviços do nível primário de assistência à saúde ${ }^{10}$.

Portanto, o acompanhamento dos casos de tuberculose pelos profissionais de saúde da família deve estar fundamentado no resgate da humanização do cuidado, por meio da escuta solidária, a partir da identificação das necessidades do doente. Desse modo, o doente torna-se mais receptivo à equipe que o acompanha $\mathrm{e}$ à terapêutica proposta. Evita-se, assim, o abandono, a ocorrência de multirresistência e facilita-se a quebra da cadeia de transmissão.

Nessa perspectiva, a VD emerge como uma estratégia que integra a valorização do cuidado especial e o reconhecimento do doente de tuberculose, ampliando as possibilidades terapêuticas, a partir da adesão e conclusão do tratamento, componentes essenciais para o controle efetivo da tuberculose e a redução de multidroga resistência.

\section{Sentimentos: segurança e gratidão no enfren- tamento da situação}

Durante a realização da visita domiciliária, emergiram sentimentos de segurança, gratidão e reconhecimento da assistência oferecida pelos profissionais da ESF, verificados nas manifestações dos usuários: 
[...] muito bem recebida, a enfermeira vinha sempre na minha casa. Ave Maria, é um ouro! (E33)

Foi sempre lá em casa providenciar a medicação, porque tem muita gente que não tem condição de chegar à unidade de saúde (E34)

Para os entrevistados, fazer o acolhimento além da assistência profissional implica conversar com as pessoas e avaliar o que elas precisam. Isso ocorre, principalmente, quando eles vivenciam momentos de conflito, como, por exemplo: piora do quadro clínico, dificuldade econômica e social, que dizem respeito às reais condições de renda e sobrevivência.

Assim, é preciso que formas inovadoras, de caráter participativo, sejam empregadas nas atividades de educação em saúde pelas equipes de saúde da família, principalmente quando se tratar de pessoas adoecidas por TB, considerando a dimensão plural da doença ${ }^{10,19}$.

Nessa perspectiva, a ESF precisa estar preparada para atender, não só às expectativas dos doentes em relação a sua doença, como também a outras expectativas ligadas ao seu grupo familiar, fatores culturais, sociais e econômicos, estabelecendo vínculos e um processo de aprendizagem mútuo, que envolve uma multiplicidade de valores, práticas, saberes e percepções de mundo ${ }^{20}$.

Entende-se, portanto, que, a partir da experiência de vida dos sujeitos adoecidos e profissionais envolvidos no cuidado, é possível articular consciência e ação, segundo a abordagem de uma educação libertadora, com sujeitos partícipes na saúde, reconhecendo que esse plano de cuidados seja eticamente respaldado no direito do cidadão à saúde.

\section{Divulgação: uma questão de dispersão da informação}

Analisando o ponto de vista do entrevistado sobre a assistência à saúde, percebe-se o interesse de se tornar cada vez mais autônomo e independente no manejo do seu processo saúde-doença, conforme exemplos dos trechos dos depoimentos a seguir:

[...] divulgar mais, conversar com as pessoas sobre a doença... Ter umas visitas nas casas, saber conversar sobre isso com as famílias. (E15)

[...] colocar gente para dar um bom treinamento, para localizar e visitar as famílias para conversar. (E16)

Além disso, os relatos dos entrevistados mostram que a educação em saúde não ocorre somente em espaços formalmente instituídos, uma vez que pode acontecer em quaisquer encontros entre aqueles que cuidam e aqueles que são cuidados, ou seja, no domicílio, espaços sociais, entre outros. Nesse contexto, o doente de tuberculose tem a oportunidade de ampliar a compreensão de seu problema e refletir a respeito da intervenção sobre a realidade em que vive, privilegiando a promoção da sua autonomia.
Em se tratando do empoderamento dos doentes por TB, como estratégia para o controle da doença, ressalta-se a importância da educação em saúde como um ato político, capaz de promover o diálogo entre profissionais de saúde e usuários, a autonomia cidadã e o incentivo a uma postura ativa desses sujeitos em seus ambientes políticos e sociais ${ }^{19,21}$. Nesse contexto, o doente de tuberculose tem a oportunidade de ampliar a compreensão de seu problema e refletir a respeito da intervenção sobre a realidade em que vive, privilegiando a promoção da sua autonomia.

Por sua história antiga, a tuberculose, nos dias atuais, ainda constitui uma doença envolta em preconceitos, fortemente associada ao contágio; porém, esse problema precisa ser melhor trabalhado pelos serviços de saúde. Tal situação demonstra a necessidade de um trabalho conjunto entre o doente de tuberculose e seu familiar.

modo como a equipe de saúde se organiza para desenvolver o seu trabalho é determinante para promover a adesão da pessoa doente ao tratamento, conduzindo-a à alta por cura. Assim, o desafio que se vislumbra no cuidado ao doente de TB, sua família e comunidade é estabelecido mediante ações de educação em saúde, relações de vínculo entre profissionais da saúde e usuários, capazes de aguçar o diálogo, a troca de saberes, o aprendizado mútuo e a participação de todos no controle da doença ${ }^{19}$.

Nesse contexto, torna-se necessária a promoção de uma prática educativa que inclua a participação ativa dos doentes de tuberculose e a família, direcionando esse trabalho de acordo com suas necessidades, crenças, representações e histórias de vida, tornandoos coprodutores desse processo educativo, juntamente com os profissionais de saúde ${ }^{13}$. Dessa forma, novas demandas podem ser identificadas pelo doente e familiar ao longo do processo de cuidado.

\section{Conclusão}

Entende-se que o processo de adoecimento do paciente com tuberculose envolve questões que ultrapassam os limites da biologia, estando relacionado a múltiplos fatores, e não apenas à relação de causa e efeito entre o bacilo e o corpo infectado.

Entretanto, observou-se no estudo um foco de atenção à saúde por parte dos profissionais da ESF ainda centrado no saber médico, praticamente inexistindo espaço para o diálogo e a construção do conhecimento. Além disso, um distanciamento dos profissionais de saúde da ESF do seu papel de educador, visto que a frequência de suas atividades educativas não condiz com a demanda apresentada pelos doentes de tuberculose.

Espera-se evidentemente que o trabalho das equipes avance no sentido de incorporar não somente a supervisão medicamentosa, como também as ações 
de diagnóstico, acompanhamento terapêutico do paciente com tuberculose, avaliação dos comunicantes e ações educativas na comunidade adstrita.

É interessante notar que a visita domiciliária traz um novo significado às práticas dos profissionais da ESF, pois essa visita é exercida junto ao indivíduo/família. Essa ação, além de estimular a partilha de experiências, integra o processo de educação em saúde e envolve, portanto, a necessidade de que os profissionais de saúde tenham um conhecimento abrangente, que vá além dos aspectos clínicos e fisiopatológicos, e que inclua a compreensão do que é viver com a tuberculose, buscando a cura.

\section{REFERÊNCIAS}

1.World Health Organization. WHO Report 2011: global tuberculosis control: surveillance, planning, financing. Geneva (Swi): WHO; 2011.

2.Clementino FS, Martiniano MS, Clementino MJSM,SousaJC,Marcolino EC, Miranda FAN.Tuberculose: desvendando conflitos pessoais e sociais. Rev enferm UERJ. 2011; 19: 638-43.

3.Ministério da Saúde (Br). Norma Operacional Básica (NOB) do SUS 1993. Brasília (DF): Ministério da Saúde; 1993.

4.Japur M, Borges CC. Sobre a (não) adesão ao tratamento: ampliando sentidos do autocuidado. Texto e contexto-enferm. 2008; 17: 64-1.

5.Figueiredo TMRM. Acesso ao tratamento de tuberculose: avaliação das características organizacionais e de desempenho dos serviços de saúde - Campina Grande/ $\mathrm{PB}$, Brasil [tese de doutorado]. Ribeirão Preto (SP): Universidade de São Paulo; 2008.

6.World Health Organization. Global tuberculosis control: surveillance, planning, financing. Geneva (Swi): WHO; 2006.

7.Ministério da Saúde(Br). Secretaria de Vigilância em Saúde. Tratamento diretamente observado (TDO) da tuberculose na atenção básica: protocolo de enfermagem. Brasília (DF): Ministério da Saúde; 2011.

8.Giacomozzi CM, Lacerda MR. A prática da assistência domiciliar dos profissionais da estratégia de saúde da família. Texto contexto-enferm. 2006; 15: 645-53.

9.Machado LC. A visita domiciliar na visão dos profissionais de saúde e dos usuários no município de Aracaju SE [dissertação de mestrado] Aracaju (SE): Universidade Tiradentes; 2010.

10.Santos TMMG, Nogueira LT, Santos LNM, Costa $\mathrm{CM}$. Acesso ao tratamento de tuberculose. Rev enferm UERJ. 2012; 20: 300-5.

11.Marcolino AB, Nogueira JA, Ruffino-Netto A, Moraes RN, Sá LD, Villa TC, et al. Evaluation of access to tuberculosis control actions in the context of family health teams in Bayeux - PB. Rev Bras Epidemiol. 2009;12:144-57. [Portuguese].

12.Silva ACO, Sousa MCM, Nogueira JA, Motta MCS. Tratamento supervisionado no controle da tuberculose: potencialidades e fragilidades na percepção do enfermeiro. Revista Eletrônica de Enf.2007 [citado em 22 set 2014]; 9: 402-16. Disponível em: http://www.facenf.uerj. $\mathrm{br} / \mathrm{v} 20 \mathrm{n} 3 / \mathrm{v} 20 \mathrm{n} 3 \mathrm{a} 17 . \mathrm{pdf}$.

13.Terra MF, Bertolozzi MR. O tratamento diretamente supervisionado (DOTS) contribui para a adesão ao tratamento da tuberculose. Rev Latino-Am Enfermagem. 2008; 16: 659-64.

14. Arakawa T, Arcêncio RA, Scatolin BE, Scatena LM, Ruffino-Netto A, Villa TCS. Acessibilidade ao tratamento de tuberculose: avaliação de desempenho de serviços de saúde. Rev Latino-Am Enfermagem [Scielo - ScientificElectronic Library Online] 2011 [citado em 23 set 2014] 19: 994-1002. Disponível em: http://www. scielo.br/pdf/rlae/v19n4/pt_19.pdf.

15.Hino P, Takahashi RF, Bertolozzi MR, Villa TCS, EgryEY.Conhecimento da equipe de saúde da família acerca das necessidades de saúde das pessoas com tuberculose. Rev Latino-Am Enfermagem [Scielo - ScientificElectronic Library Online] 2012 [citado em 23 set 2014]; 20: 44-1. Disponível em: http://www.scielo. br/pdf/rlae/v20n1/pt_07.pdf.

16.Ministério da Saúde (Br). Resolução no 196/96 de outubro de 1996. Dispõe sobre diretrizes e normas regulamentadoras de pesquisa com seres humanos. Brasília (DF): Conselho Nacional de Saúde; 1996.

17.Bardin L. Análise de conteúdo. Lisboa (Pt): Edições 70; 2009 .

18.Ministério da Saúde (Br). Secretaria de Vigilância em Saúde. Tratamento diretamente observado (TDO) da tuberculose na atenção básica: protocolo de enfermagem. Brasília (DF): Editora MS; 2011.

19.Sá LD, Gomes ALC, Carmo JB, Souza KMJ, Palha PF, Alves RS, Andrade SLE. Educação em saúde no controle da tuberculose: perspectiva de profissionais da estratégia saúde da família. Rev Eletr Enf. [Internet] 2013; 15:103-11. Disponível em: http://dx.doi.org/10. 5216/ree.v15i1.15246. doi: 10.5216/ree.v15i1.15246.

20.Alves GG, Aerts D. As práticas educativas em saúde e a estratégia saúde da família. Ciênc Saúde Coletiva [Internet]. 2011 [citado em 16 mar 2015]; 16: 319-25. Disponível em: http://www.scielosp.org/scielo.php?pid=S1413 $81232011000100034 \&$ script $=$ sci_arttext.

21.Fernandes MCP, Backes VMS. Educação em saúde: perspectivas de uma equipe da Estratégia Saúde da Família sob a óptica de Paulo Freire. Rev Bras Enferm. [Internet]. 2010 [citado em 23 abr 2015]; 63:567-73. Disponível em: http://www.scielo.br/pdf/reben/v63n4/11.pdf. 